each step of the legislative process and provided data from EPINet to support the need for such a bill.

To review a copy of this bill, go to www.senate.gov (search S 3067).

\section{Epidemiology of Fungal Infection in Neonates}

Benjamin and colleagues from the Department of Pediatrics, Duke University Medical Center, Durham, North Carolina, conducted a study to determine the epidemiology of candidemia in their neonatal intensive care unit (NICU). The study was designed to compare risk factors, clinical presentation, and outcomes for neonates infected with Candida albicans, Candida parapsilosis, and coagulase-negative staphylococcus (CNS); and to suggest a rational approach to empirical antifungal therapy of neonates at risk for nosocomial infection. Retrospective chart review of all NICU patients with systemic candidiasis or CNS infection was performed between January 1, 1995, and July 31, 1998, at Duke University Medical Center.

Fifty-one patients were reviewed. Nine of 19 patients infected with $C$ parapsilosis and 5 of 15 patients infected with $C$ albicans died of fungemia. Seventeen neonates had $>2$ positive cultures for CNS obtained within 96 hours, and 1 died. There was no statistically significant difference in birth weight, gestational age, or age at diagnosis between patient groups; however, candidemic patients had a sevenfold higher mortality rate. Before diagnosis, candidemic patients had greater exposure to systemic steroids, antibiotics, and catecholamine infusions. Of the 51 patients, 32 received thirdgeneration cephalosporins in the 2 weeks before diagnosis; 19 did not. Twenty-nine of the 32 who were treated with thirdgeneration cephalosporins subsequently developed candidemia, whereas candidemia occurred in only 5 of 19 patients who were not treated with cephalosporins. At the time of diagnosis, candidemic patients were more likely to have required mechanical ventilation and were less likely to be tolerating enteral feeding. Once the clinician was notified of a positive blood culture for Candida, patients infected with $C$ parapsilosis retained their central catheters longer than patients infected with $C$ albicans.

In this retrospective review, the authors were able to identify aspects of the clinical presentation and medication history that may be helpful in differentiating between candidemia and CNS bacteremia. Those key features may be used by clinicians to initiate empirical amphotericin B therapy in premature neonates at risk for nosocomial infections. Prolonged use of third-generation cephalosporins was strongly associated with candidemia. There was no statistically significant difference in the morbidity and mortality between patients infected with $C$ parapsilosis and those infected with $C$ albicans. Observed delays in removal of the central venous catheter may have contributed to finding a mortality rate from $C$ parapsilosis that was higher than was previously reported.

FROM: Benjamin DK, Md J, Ross K, McKinney RE, Md $\mathrm{J}$, Benjamin DK, et al. When to suspect fungal infection in neonates: a clinical comparison of Candida albicans and
Candida parapsilosis fungemia with coagulase-negative staphylococcal bacteremia. Pediatrics 2000;106:712-718.

\section{VRE: A Review}

Cetinkaya, Falk, and Mayhall from the Department of Healthcare Epidemiology and Division of Infectious Diseases, University of Texas Medical Branch at Galveston, Galveston, Texas, have written an extensive review of vancomycin-resistant enterococci (VRE). They point out that after VRE first were identified in the mid-1980s, they spread rapidly and became a major problem in many institutions in both Europe and the United States. Since VRE have intrinsic resistance to most of the commonly used antibiotics and the ability to acquire resistance to most of the current available antibiotics, either by mutation or by receipt of foreign genetic material, they have a selective advantage over other microorganisms in the intestinal flora and pose a major therapeutic challenge. The possibility of transfer of vancomycin resistance genes to other gram-positive organisms raises significant concerns about the emergence of vancomycinresistant Staphylococcus aureus. The authors review VRE, including their history, mechanisms of resistance, epidemiology, control measures, and treatment.

FROM: Cetinkaya Y, Falk P, Mayhall CG. Vancomycinresistant enterococci. Clin Microbiol Rev 2000;13:686-707.

\section{HCV Infections Among Dialysis Patients in The Netherlands}

Schneeberger and colleagues conducted a nationwide prospective survey on hepatitis $\mathrm{C}$ virus (HCV) infections among dialysis patients in The Netherlands. Patients were recruited from 34 dialysis centers and tested for antibodies and HCV RNA in 1995 and 1997. Seronegative serum samples were analyzed by reverse-transcriptase polymerase chain reaction in pools. HCV-RNA positive serum samples were genotyped and were partly sequenced.

In the first and second rounds, $67(2.9 \%)$ of 2,281 and 76 (3.4\%) of 2,286 patients were HCV-positive, respectively. Of 960 patients with paired serum samples, 35 were HCVpositive in both rounds, and $9 \mathrm{HCV}$-positive cases were newly identified in the second round. The incidence of $\mathrm{HCV}$ infection was 0.5 per 100 dialysis-years. Phylogenetic analysis revealed clustered sequences that indicated nosocomial transmission. Sixty percent of $\mathrm{HCV}$ infections, however, can be attributed to four interdependent risk factors: hemodialysis before 1992, kidney transplantation before 1994, and birth or dialysis in a foreign country. In conclusion, the prevalence of $\mathrm{HCV}$ infections in The Netherlands does not decline, and transmission within dialysis units continues. Adequate screening of $\mathrm{HCV}$ infections and strict enforcement of universal infection control practices are required.

FROM: Schneeberger PM, Keur I, van Loon A, Mortier D, de Coul KO, van Haperen AV, et al. The prevalence and incidence of hepatitis C virus infections among dialysis 